Annals of Pure and Applied Mathematics

Vol. 21, No. 1, 2020, 47-53

ISSN: 2279-087X (P), 2279-0888(online)

Published on 29 February 2020

www.researchmathsci.org

DOI: http://dx.doi.org/10.22457/apam.v21n1a6644

Annals of

Pure and Applied

Mathematics

\title{
Tripolar Fuzzy Bi ideal of a Near Ring
}

\author{
Rakshita Deshmukh ${ }^{1}$, P. Narasimha Swamy ${ }^{2}$ and B. Jyothi ${ }^{3}$ \\ ${ }^{1}$ Department of Mathematics, University College for Women, Osmania University \\ Hyderabad - 500095, Telangana, India. E-mail: rw2382@ gmail.com \\ ${ }^{2}$ Department of Mathematics, GITAM Deemed to be University \\ Hyderabad Campus - 502329, Telangana, India. E-mail: swamy.pasham@gmail.com \\ ${ }^{3}$ Department of Mathematics, University College for Women, Osmania University \\ Hyderabad - 500095, Telangana, India. E-mail: jyothireddydumbala@ gmail.com \\ Corresponding author: swamy.pasham@gmail.com
}

Received 24 January 2020; accepted 28 February 2020

Abstract. In this paper, we introduce the concept of tripolar fuzzy bi ideal of a near ring, which is a generalisation of tripolar fuzzy set, fuzzy bi ideal of a near ring. We would like to study few properties of it.

Keywords: Tripolar fuzzy set, bi-ideal, near ring

AMS Mathematics Subject Classification (2010): 03E72, 16Y30, 16 Y99

\section{Introduction}

The notion of near ring was introduced by Dickson [3] in 1905. Fuzzy ideals in near rings were studied by Zaid [1]. The concept of bi ideal for semi groups was introduced by Good and Hughes [4]. The notion of bi ideals in associative rings were introduced by Lajos and Szasz [5]. Bi ideals in near rings was given by Chelvam and Ganesan [15]. Senapati, et al. [13] studied cubic subalgebras and cubic closed ideals of B-algebras. Rafi and Venkateshwarlu [11] gave a relation on almost distributive lattices. Concept of fuzzy set introduced by Zadeh [16]. Since then many extensions have been given like intuitionistic fuzzy sets, interval valued fuzzy sets, bipolar sets and so on. Intuitionistic fuzzy sets have been introduced by Attanosov [2]. Selvam and Nagalakshmi [14] discussed fuzzy PMS ideals in PMS algebras. Nagireddy et el. [8] gave a note on fuzzy bi ideals in ternary semigroups. Senapati, et al. [12] Initiated the notion of intuitionistic fuzzifications of ideals in BG-algebras. Lee [6] introduced the concept of bipolar valued fuzzy sets. Bipolar fuzzy set is an extension of fuzzy set whose membeshipp degree range is $[-1,1]$.

The concept of tripolar fuzzy set has been introduced by Rao [7]. Tripolar fuzzy set is a generalisation of fuzzy set, intuitionistic fuzzy set and bipolar fuzzy set. He introduced the concept of tripolar fuzzy interior ideals of a gamma semi group. The tripolar concept is useful in studying the relevant, irrelevant and the implicit counter elements. Swamy, et al. [9] introduced the notion of tripolar fuzzy ideals of a near ring. In this paper we wish to introduce the concept of tripolar fuzzy bi ideal of a near ring which 
Rakshita Deshmukh, P. Narasimha Swamy and B. Jyothi

is a generalization of tripolar fuzzy set, fuzzy bi ideal of near ring. We also intend to study some of its properties. Throughout the paper $\mathrm{R}$ denotes a right near ring.

\section{Preliminaries}

Definition 2.1. [10] A Near ring (right) is a non-empty set $R$ with two binary operations "+" and "." satisfying the following axioms:

i) $(R,+)$ is a group (not necessarily abelian)

ii) $(R,$.$) is a semi group$

iii) $(y+z) x=y x+z x$ for all $x, y, z \in R$.

Definition 2.2. An ideal of a near ring $R$ is a subset $I$ of $R$ such that

i) $(I,+)$ is a normal subgroup of $(R,+)$

ii) $I R \subseteq I$

iii) $x(y+i)-x y \in I$ for all $i \in I$ and $x, y \in R$.

Definition 2.3. [15] A subgroup $I$ of $(R,+)$ satisfying $I R I \cap(I R) * I \subseteq I$ is called a bi ideal of $R$.

Definition 2.4. [16] A mapping $\mu: N \rightarrow[0,1]$ is called a fuzzy subset of $N$.

Definition 2.5. [1] A fuzzy subset $\mu$ of a near ring $R$ is called a fuzzy sub near ring of $R$ if

i) $\mu(x-y) \geq \min \{\mu(x), \mu(y)\}$,

ii) $\mu(x y) \geq \min \{\mu(x), \mu(y)\}$ for all $x, y \in R$.

Definition 2.6. A fuzzy sub near ring $\mu$ is a fuzzy ideal of a near ring $R$ if

i) $\mu(y+x-y) \geq \mu(x)$,

ii) $\mu(x y) \geq \mu(x)$

iii) $\mu(x(y+i)-x y) \geq \mu(i)$ for all $x, y, i \in R$.

Definition 2.7. A fuzzy subset $\mu$ of $R$ is called a fuzzy bi ideal of $R$, if

i) $\mu(x-y) \geq \min \{\mu(x), \mu(y)\}$,

ii) $\mu(x y z) \geq \min \{\mu(x), \mu(z)\}$.

Definition 2.8. Let $M$ and $N$ be two near rings. A mapping $f: M \rightarrow N$ is said to be a near ring homomorphism if

i) $f(x+y)=f(x)+f(y)$,

ii) $f(x y)=f(x) f(y)$ for all $x, y \in M$. 


\section{Tripolar Fuzzy Bi ideal of a Near ring}

Definition 2.9. Let $\phi: M \rightarrow N$ be a homomorphism of near rings and $f$ be a fuzzy subset of $M$. Then $\phi(f)(y)=\sup _{x \in \phi^{-1}(y)} f(x)$, if $\phi^{-1}(y) \neq 0$

$$
=0 \text {, otherwise. }
$$

We call $\phi(f)$ is the image of $f$ under $\phi$.

Definition 2.10. [6] A bipolar fuzzy set $\mu=\left(\mu^{+}, \mu^{-}\right)=\left\{\left(x, \mu^{+}(x), \mu^{-}(x)\right): x \in X\right\}$, where $\mu^{+}: X \rightarrow[0,1]$ and $\mu^{-}: X \rightarrow[-1,0]$ are membership functions. $\mu^{+}(x)$ is a degree of membership of the bipolar fuzzy set $\mu=\left\{\left(x, \mu^{+}(x), \mu^{-}(x)\right): x \in X\right\}$ and $\mu^{-}(x)$ is a degree of non-membership of the bipolar fuzzy set $\mu=\left\{\left(x, \mu^{+}(x), \mu^{-}(x)\right): x \in X\right\}$.

Definition 2.11. [7] A fuzzy subset $T$ of a universe set $X$ is said to be a tripolar fuzzy set, if

$T=\left\{\left(x, \alpha_{T}(x), \beta_{T}(x), \gamma_{T}(x)\right) / x \in X\right.$ and $\left.0 \leq \alpha_{T}(x)+\beta_{T}(x) \leq 1\right\}$, where $\alpha_{T}: X \rightarrow[0,1], \beta_{T}: X \rightarrow[0,1], \gamma_{T}: X \rightarrow[-1,0]$. The membership degree $\alpha_{T}(x)$ characterises the extent that the element $x$ satisfies to the property corresponding to tripolar fuzzy set $T, \beta_{T}(x)$ characterises the extent that the element $x$ satisfies to the not property (irrelevant) corresponding to tripolar fuzzy set $T$ and $\gamma_{T}(x)$ characterises the extent that the element $\mathrm{x}$ satisfies to the implicit counter property of tripolar fuzzy set $T$. $T=\left\{\left(x, \alpha_{T}(x), \beta_{T}(x), \gamma_{T}(x)\right) / x \in X\right.$ and $\left.0 \leq \alpha_{T}(x)+\beta_{T}(x) \leq 1\right\}$ is denoted by $T=\left(\alpha_{T}, \beta_{T}, \gamma_{T}\right)$. Thus, a tripolar fuzzy set $T$ is a generalization of fuzzy set, bipolar fuzzy set and intuitionistic fuzzy set.

Definition 2.12. [9] A tripolar fuzzy set $T=\left(\alpha_{T}, \beta_{T}, \gamma_{T}\right)$ of a near ring $R$ is called a tripolar fuzzy sub near ring of $R$ if it satisfies the following conditions:

i) $\alpha_{T}(x-y) \geq \min \left\{\alpha_{T}(x), \alpha_{T}(y)\right\}$,

ii) $\alpha_{T}(x y) \geq \min \left\{\alpha_{T}(x), \alpha_{T}(y)\right\}$,

iii) $\beta_{T}(x-y) \leq \max \left\{\beta_{T}(x), \beta_{T}(y)\right\}$,

iv) $\beta_{T}(x y) \leq \max \left\{\beta_{T}(x), \beta_{T}(y)\right\}$,

v) $\gamma_{T}(x-y) \leq \max \left\{\gamma_{T}(x), \gamma_{T}(y)\right\}$

vi) $\gamma_{T}(x y) \leq \max \left\{\gamma_{T}(x), \gamma_{T}(y)\right\}$.

Definition 2.13. [9] A tripolar fuzzy sub near ring $T=\left(\alpha_{T}, \beta_{T}, \gamma_{T}\right)$ of a near ring $R$ is called a tripolar fuzzy ideal of $R$ if $T$ satisfies the following conditions:

i) $\alpha_{T}(x y) \geq\left\{\alpha_{T}(x)\right\}$, 
Rakshita Deshmukh, P. Narasimha Swamy and B. Jyothi

ii) $\alpha_{T}(x(y+i)-x y) \geq\left\{\alpha_{T}(i)\right\}$,

iii) $\alpha_{T}(y+x-y) \geq\left\{\alpha_{T}(x)\right\}$,

iv) $\beta_{T}(y+x-y) \leq\left\{\beta_{T}(x)\right\}$,

v) $\beta_{T}(x y) \leq\left\{\beta_{T}(x)\right\}$,

vi) $\beta_{T}(x(y+i)-x y) \leq\left\{\beta_{T}(i)\right\}$,

vii) $\gamma_{T}(y+x-y) \leq\left\{\gamma_{T}(x)\right\}$,

viii) $\gamma_{T}(x y) \leq\left\{\gamma_{T}(x)\right\}$,

ix) $\gamma_{T}((x+i) y-x y) \leq\left\{\gamma_{T}(i)\right\}$ for all $x, y, i \in R$.

\section{Tripolar fuzzy bi ideal}

In this section we introduce the concept of tripolar fuzzy bi ideal and study some of their properties.

Definition 3.1. A tripolar fuzzy set $T=\left(\alpha_{T}, \beta_{T}, \gamma_{T}\right)$ of a near ring $R$ is called a tripolar fuzzy bi ideal of $R$, if

i) $\quad \alpha_{T}(x-y) \geq \min \left\{\alpha_{T}(x), \alpha_{T}(y)\right\}$

ii) $\alpha_{T}(x y z) \geq \min \left\{\alpha_{T}(x), \alpha_{T}(z)\right\}$,

iii) $\beta_{T}(x-y) \leq \max \left\{\beta_{T}(x), \beta_{T}(y)\right\}$,

iv) $\beta_{T}(x y z) \leq \max \left\{\beta_{T}(x), \beta_{T}(z)\right\}$,

v) $\gamma_{T}(x-y) \leq \max \left\{\gamma_{T}(x), \gamma_{T}(y)\right\}$,

vi) $\gamma_{T}(x y z) \leq \max \left\{\gamma_{T}(x), \gamma_{T}(z)\right\}$.

for every $x, y \in R$.

Example 3.1. Let $R=\{0, a, b, c\}$ be a near ring with the binary operations defined below:

\begin{tabular}{|c|c|c|c|c|}
\hline+ & 0 & $a$ & $b$ & $c$ \\
\hline 0 & 0 & $a$ & $b$ & $c$ \\
\hline$a$ & $a$ & 0 & $c$ & $b$ \\
\hline$b$ & $b$ & $c$ & 0 & $a$ \\
\hline$c$ & $c$ & $b$ & $a$ & 0 \\
\hline
\end{tabular}

\begin{tabular}{|l|l|l|l|l|}
\hline$\bullet$ & 0 & $a$ & $b$ & $c$ \\
\hline 0 & 0 & 0 & 0 & 0 \\
\hline$a$ & 0 & 0 & $a$ & $a$ \\
\hline$b$ & 0 & 0 & $b$ & $b$ \\
\hline$c$ & 0 & 0 & $c$ & $c$ \\
\hline
\end{tabular}

Define a tripolar fuzzy set $T=\left(\alpha_{T}, \beta_{T}, \gamma_{T}\right)$ by $T=\{(0,0.8,0.2,-0.4),(a, 0.6,0.3,-0.2),(b, 0.6,0.3,-0.2),(c, 0.6,0.3,-0.2)\}$. Then $T$ is a tripolar fuzzy bi-ideal of the near $\operatorname{ring} R$. 


\section{Tripolar Fuzzy Bi ideal of a Near ring}

Theorem 3.1. Intersection of two tripolar fuzzy bi ideals of $\mathrm{R}$ is also its tripolar fuzzy bi ideal.

Proof: Let $f=\left(\alpha_{f}, \beta_{f}, \gamma_{f}\right), g=\left(\alpha_{g}, \beta_{g}, \gamma_{g}\right)$ be two tripolar fuzzy bi ideals of $\mathrm{R}$ and $x, y \in R$. Then

$$
\begin{aligned}
\begin{aligned}
\alpha_{f \cap g}(x-y) & =\min \left(\alpha_{f}(x-y), \alpha_{g}(x-y)\right) \geq \min \left(\min \left(\alpha_{f}(x), \alpha_{f}(y)\right), \min \left(\alpha_{g}(x), \alpha_{g}(y)\right)\right) \\
& =\min \left(\min \left(\alpha_{f}(x), \alpha_{g}(x)\right), \min \left(\alpha_{f}(y), \alpha_{g}(y)\right)\right)=\min \left(\alpha_{f \cap g}(x), \alpha_{f \cap g}(y)\right), \\
\alpha_{f \cap g}(x y z)= & \min \left(\alpha_{f}(x y z), \alpha_{g}(x y z)\right) \geq \min \left(\min \left(\alpha_{f}(x), \alpha_{f}(z)\right), \min \left(\alpha_{g}(x), \alpha_{g}(z)\right)\right) \\
= & \min \left(\min \left(\alpha_{f}(x), \alpha_{g}(x)\right), \min \left(\alpha_{f}(z), \alpha_{g}(z)\right)\right)=\min \left(\alpha_{f \cap g}(x), \alpha_{f \cap g}(z)\right), \\
\beta_{f \cap g}(x-y) & =\max \left(\beta_{f}(x-y), \beta_{g}(x-y)\right) \geq \max \left(\max \left(\beta_{f}(x), \beta_{f}(y)\right), \max \left(\beta_{g}(x), \beta_{g}(y)\right)\right) \\
& =\max \left(\max \left(\beta_{f}(x), \beta_{g}(x)\right), \max \left(\beta_{f}(y), \beta_{g}(y)\right)\right)=\max \left(\beta_{f \cap g}(x), \beta_{f \cap g}(y)\right), \\
\beta_{f \cap g}(x y z)= & \max \left(\beta_{f}(x y z), \beta_{g}(x y z)\right) \geq \max \left(\max \left(\beta_{f}(x), \beta_{f}(z)\right), \max \left(\beta_{g}(x), \beta_{g}(z)\right)\right) \\
= & \max \left(\max \left(\beta_{f}(x), \beta_{g}(x)\right), \max \left(\beta_{f}(z), \beta_{g}(z)\right)\right)=\max \left(\beta_{f \cap g}(x), \beta_{f \cap g}(z)\right), \\
\gamma_{f \cap g}(x-y) & =\max \left(\gamma_{f}(x-y), \gamma_{g}(x-y)\right) \geq \max \left(\max \left(\gamma_{f}(x), \gamma_{f}(y)\right), \max \left(\gamma_{g}(x), \gamma_{g}(y)\right)\right) \\
& =\max \left(\max \left(\gamma_{f}(x), \gamma_{g}(x)\right), \max \left(\gamma_{f}(y), \gamma_{g}(y)\right)\right)=\max \left(\gamma_{f \cap g}(x), \gamma_{f \cap g}(y)\right), \\
\gamma_{f \cap g}(x y z)= & \max \left(\gamma_{f}(x y z), \gamma_{g}(x y z)\right) \geq \max \left(\max \left(\gamma_{f}(x), \gamma_{f}(z)\right), \max \left(\gamma_{g}(x), \gamma_{g}(z)\right)\right) \\
= & \max \left(\max \left(\gamma_{f}(x), \gamma_{g}(x)\right), \max \left(\gamma_{f}(z), \gamma_{g}(z)\right)\right)=\max \left(\gamma_{f \cap g}(x), \gamma_{f \cap g}(z)\right) .
\end{aligned}
\end{aligned}
$$

Hence proved.

Theorem 3.2. If a tripolar fuzzy set $T=\left(\alpha_{T}, \beta_{T}, \gamma_{T}\right)$ of near $\operatorname{ring} R$ is a tripolar fuzzy bi- ideal of $R$, then $\left(\alpha_{T}, \bar{\alpha}_{T}, \gamma_{T}\right)$, where $\bar{\alpha}_{T}=1-\alpha_{T}$ is a tripolar fuzzy bi ideal of $R$.

Proof: Let $x, y, z \in R$. Then $\bar{\alpha}_{T}(x-y)=1-\alpha_{T}(x-y) \leq 1-\min \left\{\alpha_{T}(x), \alpha_{T}(y)\right\}$

$$
=\max \left\{1-\alpha_{T}(x), 1-\alpha_{T}(y)\right\}=\max \left\{\bar{\alpha}_{T}(x), \bar{\alpha}_{T}(y)\right\} \text { and } \bar{\alpha}_{T}(x y z)=1-\alpha_{T}(x y z)
$$$$
\leq 1-\min \left\{\alpha_{T}(x), \alpha_{T}(z)\right\}=\max \left\{1-\alpha_{T}(x), 1-\alpha_{T}(z)\right\}=\max \left\{\overline{\alpha_{T}}(x), \overline{\alpha_{T}}(z)\right\} .
$$

Theorem 3.3. Let $f: M \rightarrow N$ be a near ring homomorphism. If $T=\left(\alpha_{T}, \beta_{T}, \gamma_{T}\right)$ is a tripolar fuzzy bi-ideal of $N$, then $f^{-1}(T)=\left(f^{-1}\left(\alpha_{T}\right), f^{-1}\left(\beta_{T}\right), f^{-1}\left(\gamma_{T}\right)\right)$ is a tripolar fuzzy bi ideal of $M$.

Proof: Let $x, y \in M$.

$$
\begin{aligned}
f^{-1}\left(\alpha_{T}(x-y)\right) & =\alpha_{T}(f(x-y)) \\
& =\alpha_{T}(f(x)-f(y)) \\
& \geq \min \left\{\alpha_{T}(f(x)), \alpha_{T}(f(y))\right\} \\
& =\min \left\{f^{-1}\left(\alpha_{T}(x)\right), f^{-1}\left(\alpha_{T}(y)\right)\right\},
\end{aligned}
$$


Rakshita Deshmukh, P. Narasimha Swamy and B. Jyothi

$$
\begin{aligned}
f^{-1}\left(\alpha_{T}(x y z)\right) & =\alpha_{T}(f(x y z)) \\
& =\alpha_{T}(f(x) f(y) f(z)) \\
& \geq \min \left\{\alpha_{T}(f(x)), \alpha_{T}(f(z))\right\} \\
& =\min \left\{f^{-1}\left(\alpha_{T}(x)\right), f^{-1}\left(\alpha_{T}(z)\right)\right\}, \\
f^{-1}\left(\beta_{T}(x-y)\right) & =\beta_{T}(f(x-y)) \\
& =\beta_{T}(f(x)-f(y)) \\
& \leq \max \left\{\beta_{T}(f(x)), \beta_{T}(f(y))\right\} \\
& =\max \left\{f^{-1}\left(\beta_{T}(x)\right), f^{-1}\left(\beta_{T}(y)\right)\right\}, \\
f^{-1}\left(\beta_{T}(x y z)\right) & =\beta_{T}(f(x y z)) \\
& =\beta_{T}(f(x) f(y) f(z)) \\
& \leq \max \left\{\beta_{T}(f(x)), \beta_{T}(f(z))\right\} \\
& =\max \left\{f^{-1}\left(\beta_{T}(x)\right), f^{-1}\left(\beta_{T}(z)\right)\right\}, \\
f^{-1}\left(\gamma_{T}(x-y)\right) & =\gamma_{T}(f(x-y)) \\
& =\gamma_{T}(f(x)-f(y)) \\
& \leq \max \left\{\gamma_{T}(f(x)), \gamma_{T}(f(y))\right\} \\
& =\max \left\{f-1\left(\gamma_{T}(x)\right), f^{-1}\left(\gamma_{T}(y)\right)\right\}, \\
f^{-1}\left(\gamma_{T}(x y z)\right) & =\gamma_{T}(f(x y z)) \\
& =\gamma_{T}(f(x) f(y) f(z)) \\
& \leq \max \left\{\gamma_{T}(f(x)), \beta_{T}(f(z))\right\} \\
& =\max \left\{f^{-1}\left(\gamma_{T}(x)\right), f^{-1}\left(\gamma_{T}(z)\right)\right\} .
\end{aligned}
$$

Thus $f^{-1}(T)=\left(f^{-1}\left(\alpha_{T}\right), f^{-1}\left(\beta_{T}\right), f^{-1}\left(\gamma_{T}\right)\right)$ is a tripolar fuzzy bi ideal of $M$.

Acknowledgment: The authors would like to thank Prof. Madhumangal Pal, Editor in Chief and also very much grateful to the referees for their valuable comments and suggestions for improving this paper.

\section{REFERENCES}

1. S.Abou Zaid, On fuzzy sub near-rings and ideals, Fuzzy Sets and Systems, 44 (1991) 139-146.

2. K.T.Attanosov, Intuitionistic fuzzy sets, Fuzzy Sets and Systems, 20 (1) (1986) 87-96.

3. L.E.Dickson. On finite algebras, Nachrichten von der Gesellschaft der Wissenschaften zu Göttingen, Mathematishch-Physikalische Klasse, 1905 (1905) 358-393.

4. R.H.Good and D.R.Hughes, Associated groups for semigroup, Bull. Amer. Math. Soc., 58 (1952) 624-625.

5. S.Lajos and F.Szasz, Bi ideals in associative rings, Acta. Sci. Math. Szeged, 32 (1971) 185-193. 


\section{Tripolar Fuzzy Bi ideal of a Near ring}

6. K.M.Lee, Bipolar valued fuzzy sets and their operations, Proc. Int. Conf. on Intelligent Technologies, Bangkok, Thailand, (2000) 307-312.

7. M.Murali Krishna Rao, Tripolar fuzzy interior ideals of gamma semigroup, Annals of Fuzzy Mathematics and Informatics, 15(2) (2018) 199-206.

8. U.Nagi Reddy, K.Rajani and G.Shobhalatha, A note on fuzzy bi ideals in ternary semigroups, Annals of Pure and Applied Mathematics, 16(2) (2018) 295-304.

9. P.N.Swamy, R.Deshmukh, G.N.Rao and T.Srinivas, Tripolar fuzzy ideals of a near ring, Iranian Journal of Fuzzy Systems, Communicated.

10. G.Pilz, Near-ring, North Holland, Amsterdam, (1983).

11. N.Rafi and B.Venkateshwarlu, A relation on almost distributive lattices, Annals of Pure and Applied Mathematics, 2(2) (2012) 129-134.

12. T.Senapati, M Bhowmik and M.Pal, Intuitionistic fuzzifications of ideals in BGalgebras, Mathematica Aeterna, 2(9) (2015) 761-778.

13. T.Senapati, C.S.Kim, M Bhowmik and M.Pal, Cubic subalgebras and cubic closed ideals of B-algebras, Fuzzy Information and Engineering, 7(2) (2015) 129-149.

14. P.M.Sithar Selvam and K.T.Naga Lakshmi, Fuzzy PMS ideals in PMS algebras, Annals of Pure and Applied Mathematics, 12(2) (2016) 153-159.

15. T.Tamiz Chelvam and N.Ganesan, On bi ideals of near ring, Indian Journal of Pure and Applied Mathematics, 18(11) (1987) 1002-1005.

16. L.A.Zadeh, Fuzzy sets, Inform. Control, 8 (1965) 338-353. 